\title{
Melatonin reduced volume of cerebral infarct induced by photothrombosis in wild-type mice, not in Cyclooxygenase-1 gene knockout mice
}

\author{
L.Y. Zou ${ }^{1}$, S.R. Liu ${ }^{2}$, G. Li ${ }^{3}$, L. Huang ${ }^{2}$, Edward S. Yang ${ }^{3}$ \\ ${ }^{1}$ Department of Medicine, The University of Hong Kong, Hong Kong \\ ${ }^{2}$ Imaging Center of the First Affiliated Hospital of Medical College, JinanUniversity, China \\ ${ }^{3}$ The Jockey Club Magnetic Resonance Imaging Engineering Centre, The University of Hong Kong, Hong Kong
}

\begin{abstract}
Cyclooxygenase (COX) is crucial in inflammation and plays important role in cerebral ischemia. Antiinflammatory effects of melatonin have been verified in previous studies. In this study, cerebral blood flow (CBF) was monitored during operation, infarct volume (IFV) was determined with 5-triphenyltetrazolium chloride (TTC) staining and MR image, and neurological functions were evaluated with turn in an alley and fall pole test in both COX1-gene knockout and wide-type mice with or without melatonin administration 3 days after photothrombosis. CBF reduction, IFV and neurological deficits were not significantly different in COX-1 wild-type and COX-1 knockout mice. Melatonin (15 $\mathrm{mg} / \mathrm{kg}$ ) intraperitoneal injection decreased the $\mathrm{CBF}$ reduction, IFV and the latency to turn in an alley in COX-1 wide-type mice, whereas the neuroprotective effect of melatonin was attenuated in COX-1 knockout mice. We concluded that melatonin reduced susceptibility to photothrombotic stroke. COX-1 gene knockout does not alter the susceptibility to cerebral ischemia caused by photothrombosis. COX-1 plays an important role in the pathway of the protection of melatonin.
\end{abstract}

Keywords - Cyclooxygenase-1 knockout, cerebral infarct, melatonin, MR image

\section{INTRODUCTION}

Stroke is the third leading cause of death and a major source of disability, and ischemic stroke is the most common type. COX contributes to the delayed ischemic damage via inflammation. There are two isoforms: COX-1 and COX-2. COX-1 is constitutively expressed and responsible for many physiological functions, however, the role of COX-1 in cerebral ischemia is not well understood. In the middle cerebral artery occlusion (MCAO) mouse stroke model, Cheung RT et. al found that susceptibility to ischemia did not alter in COX-1 gene knockout mice [1], while Iadecola $\mathrm{C}$ showed susceptibility increased in COX-1deficient mice [2]. Melatonin has been shown to possess marked anti-inflammatory [3] and anti-carcinogenic properties [4], it can enhance the anti-inflammatory effect of non-selective COX-1 and COX-2 inhibitor indomethacin [5]. Melatonin also reduced volume of cerebral infarction in a rat MCAO stroke model [6]. However, the pathway of the neuroprotective effect of melatonin on cerebral ischemia is not well known. In this study, our goal is to test whether melatonin protects brain after cerebral ischemia via COX-1.

\section{Methodology}

All experiments were conducted according to the institutional guidelines with the protocol approved by the Committee on the Use of Live Animals in Teaching and Research, the University of Hong Kong. Heterozygous (COX-1-gene +/-) knockout mice of strain C57/BL were established at the National Institute of Environmental Health Science, NC [7], and a colony was set up in the Laboratory Animal Unit, University of Hong Kong. Mice were kept under a diurnal lighting condition (12 h light) with food and water provided ad libitum. A standard protocol of polymerase chain reaction (PCR) was used to determine the COX-1 genotype when the mice were 4 weeks old [1].

Induction of focal cerebral ischemia: Rose Bengal was injected intravenously before illumination, Photothrombosis was induced at $2 \mathrm{~mm}$ posterior and $3 \mathrm{~mm}$ laterally from bregma on the left side with illumination of a cold white light for $15 \mathrm{~min}$ [8].

Determination of cerebral blood flow (CBF) in penumbra: A laser Doppler flowmeter was used to monitor the regional $\mathrm{CBF}$ for $15 \mathrm{~min}$ during illumination at $6 \mathrm{~mm}$ lateral and $2 \mathrm{~mm}$ posterior to the bregma on the left side [1].

Drug treatment: Melatonin $(15 \mathrm{mg} / \mathrm{kg})$ or its vehicle was given via an intraperitoneal injection at $0.5 \mathrm{~h}$ before stroke, $24 \mathrm{~h}$ and $48 \mathrm{~h}$ after photothrombosis.

Neurological function evaluation: Neurological function assessment was performed $1 \mathrm{~h}$ before photothrombosis and $72 \mathrm{~h}$ after photothrombosis [9].

Turning in an alley was used as a measure of coordinated muscle control. The experimental animal was placed facing the back wall of an alley. The amount of time required for the animal to turn around and face the open end of the alley was recorded.

Locomotor Balance and Coordination was measured by fall pole test. The experimental animal was placed at the center of a horizontal wooden pole that was elevated $75 \mathrm{~cm}$ above the substrate. A large pillow was placed under the screen. Latency to fall was recorded.

Determination of infarct volume: IFV was evaluated at 3 days or 7 days after photothrombosis immediately following the evaluation of neurological function with 2,3,5triphenyltetrazolium chloride (TTC) staining from bregma $3.5 \mathrm{~mm}$ to bregma $-4.5 \mathrm{~mm}$. The hemispheric volumes and total infarction were integrated from the respective calibrated area measurements that were obtained from the digitized images of the TTC-stained brain slices, using a 
computer-assisted image analysis system. The infarct volumes were normalized and expressed as a percentage of the ipsilateral hemispheric volume.

$M R$ image: The Cerebral infarct volume of formalinperfused fixed mice brain was also evaluated with MR image. All MR image studies were done in a 1.5 T GE MR system at the imaging Center of Hua Qiao Hospital. Infarct volume $\left(\mathrm{mm}^{3}\right)$ was calculated by manually delineating hyperintense areas from the $\mathrm{T}_{2}$-weighted imaging by using SMIS image software. Results are expressed as lesion volume percentage from the volume of the ipsilateral hemisphere [10].

\section{RESULTS}

Melatonin $(15 \mathrm{mg} / \mathrm{kg})$ decreased the CBF in penumbra during operation in wild-type (COX -/-) mice compared with vehicle group, while in COX-1 knockout (COX-1 +/or $\mathrm{COX}-1+/+)$ mice, melatonin failed to influent the $\mathrm{CBF}$ in penumbra (Fig 1). In vehicle groups, there were no significant differences in $\mathrm{CBF}$ in penumbra among the three genotypes groups.
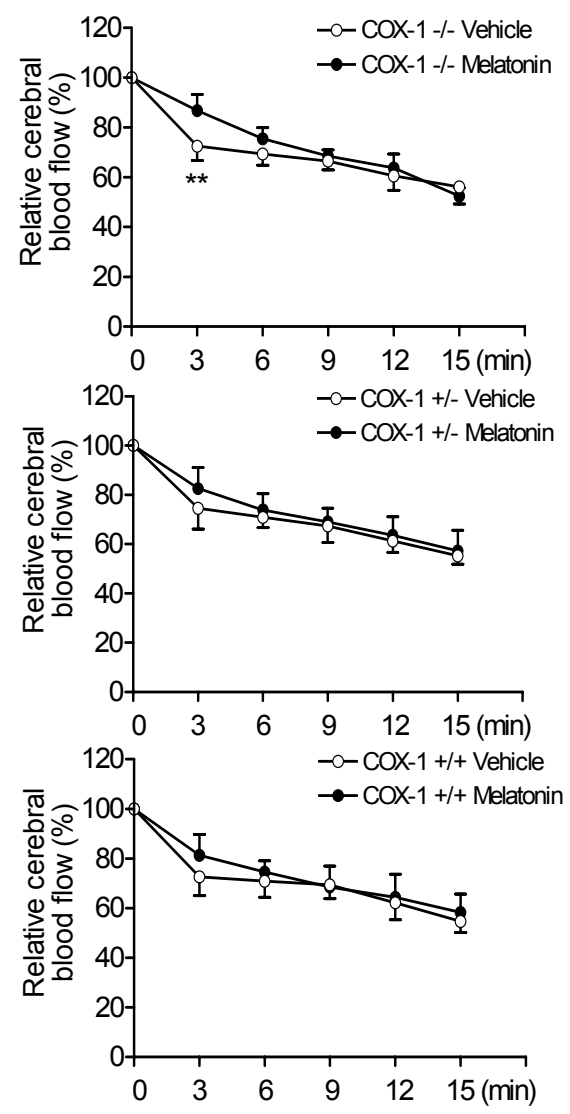

Fig 1. Effect of melatonin $(15 \mathrm{mg} / \mathrm{kg})$ on $\mathrm{CBF}$ in penumbra in the COX-1 wild type (COX-1 -/-), COX-1 knockout $(\mathrm{COX}-1+/-$ or COX $-1+/+)$ mice $(\mathrm{n}=6)$. ** $P<0.01$, compared with vehicle group
As shown in Fig 2, melatonin $(15 \mathrm{mg} / \mathrm{kg})$ reduced the relative IFV (measure with TTC staining) significantly in wild-type (COX-1 -/-) mice 3 days after photothrombosis compared with vehicle group. In COX-1 knockout (COX-1 $+/$ - or COX-1 +/+) mice, melatonin administration did not affect IFV significantly. Compared with wild type group, the IFV of COX-1 knockout groups did not change in vehicle groups.

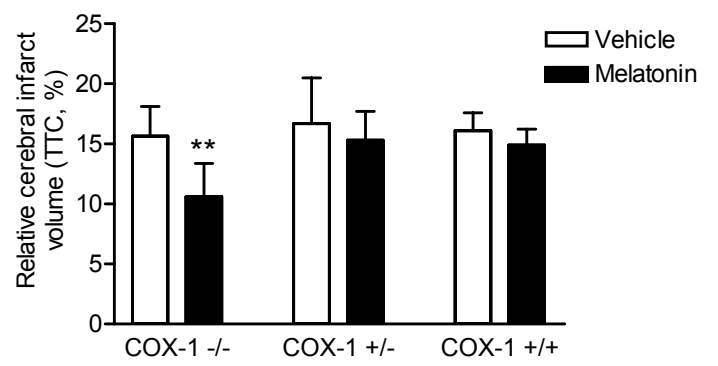

Fig 2. Effect of melatonin on IFV 3 days after photothrombosis in the COX-1 wild type (COX-1 -/), COX-1 knockout (COX-1 +/- or COX-1 +/+) mice with TTC staining $(n=6) . * * P<0.01$, compared with vehicle group

As shown in Fig 3 (A), IFV in melatonin treated group decreased in wild type mice 3 days after photothrombosis. In COX-1 knockout mice, IFV was not significant difference between melatonin treated and vehicle groups. Among three genotypes group, there were no significant differences in IFV in vehicle groups.

A

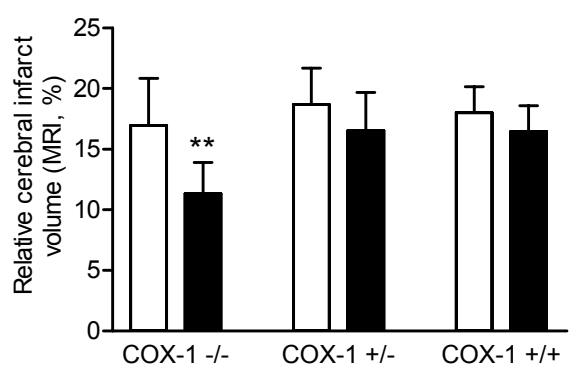

B

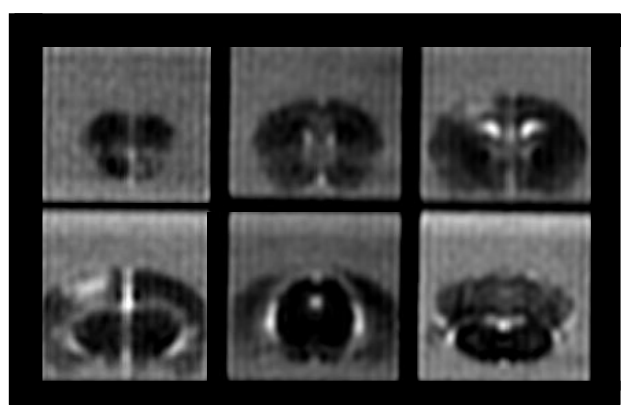

Fig 3. (A) The effect of melatonin on relative cerebral IFV 3 days after photothrombosis in the COX-1 wild type (COX-1 -/-), COX-1 knockout (COX-1 +/- or COX-1 +/+) mice $(\mathrm{n}=6 ; * * \quad P<0.01)$ compared with vehicle group. (B) MR T2-weighted images taken 3 days after photothrombosis in the COX-1 knockout $(\mathrm{COX}-1+/+)$ mice. 
As shown in Fig 4, latency to turn in an alley prolonged significantly 3 days after photothrombosis in three genotype mice groups (vehicle) compared with sham group. Melatonin $(15 \mathrm{mg} / \mathrm{kg})$ administration reduced latency to turn in an alley in wild-type (COX-1 -/-) mice group compared with vehicle group, but in COX-1 knockout (COX-1 +/- or COX $-1+/+)$ mice, melatonin had no any effects. There were no differences in latency to fall pole in sham, vehicle and treatment groups either in wild type or COX-1 knockout mice (data not shown).

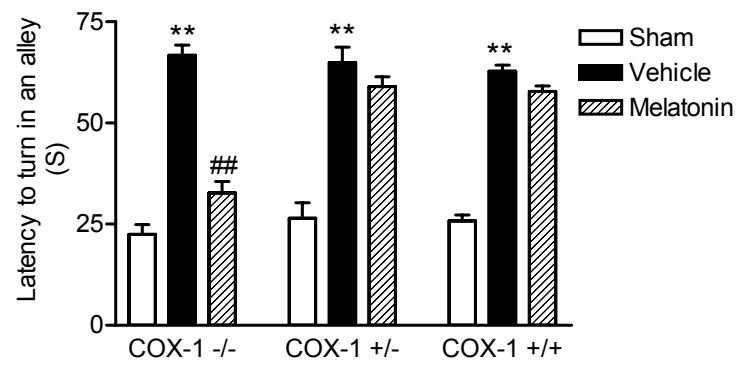

Fig 4. Effect of melatonin on latency to turn in an alley 3 days after photothrombosis in the COX-1 wild-type (COX1 -/-), COX-1 knockout (COX-1 +/- or COX-1 +/+) mice $(\mathrm{n}=6)$. ${ }^{* *} P<0.01$, compared with sham group; \#\# $P<0.01$, compared with vehicle group

\section{DISCUSSION}

Inflammation has been considered as one of the most important mechanisms upon the damage after ischemia. Anti-inflammation has been shown to be the main roles of melatonin, which is regard as the mechanism of neuroprotection [5]. The neuroprotective effect of melatonin has been reported in rat with MCAO model [6]. In the present study, the infarct volume decreased after administration of melatonin in COX-1 wild-type (COX-1 -/) mice with photothrombosis model. Previous study showed that melatonin enhanced the effect of indomethacin, a nonselective COX-1 and COX-2 inhibitor [5], but which subtype of COX is involved is still unclear.

It is controversial that whether COX-1-gene deficiency increases susceptibility to ischemia brain damage in MCAO mice model $[1,2]$. Our present study demonstrated that COX-1-gene knockout did not increase vulnerability to cerebral ischemia in $\mathrm{CBF}$, IFV and neurological functions, which is consistent with our previous results in MCAO model [1].

The most important results of the present study is that the neuroprotective effect of melatonin attenuated in COX1-gene knockout (COX-1 -/+ or COX-1 +/+) mice, implicating that $\mathrm{COX}-1$ may play a key role in the signal transduction pathway of neuroprotection of melatonin. The mechanism need further study.

\section{CONCLUSION}

Melatonin reduces susceptibility to photothrombotic stroke with improved neurological functions and decreased IFV evaluated with both TTC staining and MR images. COX-1 gene knockout does not alter the susceptibility to cerebral ischemia caused by photothrombosis. COX-1 plays an important role in the pathway of the protection of melatonin.

\section{ACKNOWLEDGMENT}

We thank Prof. T.M. Wong for use of his lab and Mr. C.P. Mok for his technical assistance.

\section{REFERENCES}

[1] Cheung, R. T., Pei, Z., Feng, Z. H., and Zou, L. Y., "Cyclooxygenase-1 gene knockout does not alter middle cerebral artery occlusion in a mouse stroke model," Neurosci.Lett., vol. 330, no. 1, pp. 57-60, Sept.2002.

[2] Iadecola, C., Sugimoto, K., Niwa, K., Kazama, K., and Ross, M. E., "Increased susceptibility to ischemic brain injury in cyclooxygenase-1-deficient mice," J.Cereb.Blood Flow Metab, vol. 21, no. 12, pp. 1436-1441, Dec.2001

[3] Cuzzocrea, S., Zingarelli, B., Gilad, E., Hake, P., Salzman, A. L., and Szabo, C., "Protective effect of melatonin in carrageenan-induced models of local inflammation: relationship to its inhibitory effect on nitric oxide production and its peroxynitrite scavenging activity," J.Pineal Res., vol. 23, no. 2, pp. 106-116, Sept.1997.

[4] Lissoni, P., Tancini, G., Paolorossi, F., Mandala, M., Ardizzoia, A., Malugani, F., Giani, L., and Barni, S., "Chemoneuroendocrine therapy of metastatic breast cancer with persistent thrombocytopenia with weekly low-dose epirubicin plus melatonin: a phase II study," J.Pineal Res., vol. 26, no. 3, pp. 169-173, Apr.1999.

[5] El Shenawy, S. M., Abdel-Salam, O. M., Baiuomy, A. R., El Batran, S., and Arbid, M. S., "Studies on the anti-inflammatory and anti-nociceptive effects of melatonin in the rat," Pharmacol.Res., vol. 46, no. 3, pp. 235-243, Sept.2002.

[6] Pei, Z., Pang, S. F., and Cheung, R. T., "Pretreatment with melatonin reduces volume of cerebral infarction in a rat middle cerebral artery occlusion stroke model," J.Pineal Res., vol. 32, no. 3, pp. 168-172, Apr.2002.

[7] Langenbach, R., Morham, S. G., Tiano, H. F., Loftin, C. D., Ghanayem, B. I., Chulada, P. C., Mahler, J. F., Lee, C. A., Goulding, E. H., Kluckman, K. D., and ., "Prostaglandin synthase 1 gene disruption in mice reduces arachidonic acidinduced inflammation and indomethacin-induced gastric ulceration," Cell, vol. 83, no. 3, pp. 483-492, Nov.1995.

[8] Schroeter, M., Jander, S., and Stoll, G., "Non-invasive induction of focal cerebral ischemia in mice by photothrombosis of cortical microvessels: characterization of inflammatory responses," J.Neurosci.Methods, vol. 117, no. 1, pp. 43-49, May2002.

[9] Hattori, K., Lee, H., Hurn, P. D., Crain, B. J., Traystman, R. J., and DeVries, A. C., "Cognitive deficits after focal cerebral ischemia in mice," Stroke, vol. 31, no. 8, pp. 1939-1944, Aug.2000.

[10] Koistinaho, M., Kettunen, M. I., Holtzman, D. M., Kauppinen, R. A., Higgins, L. S., and Koistinaho, J., "Expression of human apolipoprotein E downregulates amyloid precursor proteininduced ischemic susceptibility," Stroke, vol. 33, no. 7, pp. 1905-1910, July2002. 